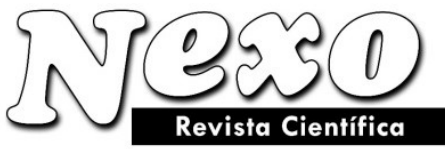

ISSN-E 1995-9516

Universidad Nacional de Ingeniería http://revistas.uni.edu.ni/index.php/Nexo http://dx.doi.org/10.5377/nexo.v30i2.5526

\title{
INFLUENCIA DEL MUCÍLAGO DE NOPAL Y EXTRACTO DE ALGAS CAFÉS EN EL GRADO DE HIDRATACIÓN DE PASTAS DE CEMENTO PORTLAND ORDINARIO
}

\section{INFLUENCE OF CACTUS MUCILAGE AND BROWN SEAWEED EXTRACT ON THE DEGREE OF HYDRATION OF ORDINARY PORTLAND CEMENT PASTES}

\author{
E.F. Hernández ${ }^{1, *}$, H. Pfeiffer Perea ${ }^{2}$, P.F. de J. Cano Barrita ${ }^{3}$ \\ ${ }^{1}$ Universidad Nacional de Ingeniería. Dirección de Investigación. Managua, Nicaragua. \\ ${ }^{2}$ Instituto de Investigaciones en Materiales, Universidad Nacional Autónoma de México, Circuito \\ Exterior, Ciudad Universitaria, Coyoacán, Ciudad de México, C.P. 04510, México. \\ ${ }^{3}$ Centro Interdisciplinario de Investigación para el Desarrollo Integral Regional, Unidad Oaxaca, Instituto \\ Politécnico Nacional, Hornos No. 1003, Sta Cruz Xoxocotlán, Oaxaca, C.P. 71230, México \\ *edifh28@hotmail.com
}

(recibido/received: 25-Septiembre-2017; aceptado/accepted: 30-Octubre-2017)

\section{RESUMEN}

Este trabajo presenta el efecto que produce la adición de dispersión acuosa de mucílago de nopal y el extracto de algas marinas cafés en el grado de hidratación de pastas de cemento Portland ordinario. Se elaboraron pastas de cemento con relaciones agua/cemento de 0.30 y 0.60 , conteniendo los aditivos naturales en tres concentraciones. Las pastas se curaron en húmedo y selladas. Se determinó el grado de hidratación de las pastas ignición y análisis termo-gravimétrico a los 14, 28 y 56 días de edad. Los resultados indican un mayor grado de hidratación en las pastas de cemento con relación agua/cemento de 0.60 conteniendo los aditivos naturales en comparación con el control. Se sugiere que esto es debido a una mejor dispersión de las partículas de cemento en la presencia de estos aditivos y a la capacidad de estos para retener agua, con lo cual se produce curado interno.

Palabras claves: Mucílago de nopal; Extracto de algas cafés; Grado de hidratación; Análisis termogravimétrico.

\begin{abstract}
This paper presents the effect produced by the addition of aqueous dispersions of cactus mucilage and brown seaweed extract on the degree of hydration of ordinary Portland cement pastes. Cement pastes with water/cement ratios of 0.30 and 0.60 were prepared containing the natural additives at three concentrations. The curing conditions were moist cured and sealed. The degree of hydration was determined by ignition and thermo-gravimetric analysis at 14, 28 and 56 days of age. The results indicate a higher degree of hydration in pastes with a water/cement ratio of 0.60 containing the natural additives with respect to control specimens. This effect could be explained by a better dispersion of cement particles in the presence of the natural additives and their capacity to retain water, thereby providing internal curing.
\end{abstract}

Keywords: Cactus mucilage; Brown seaweed extract; Degree of hydration; Thermogravimetric analysis. 


\section{INTRODUCCIÓN}

Debido a los problemas de durabilidad en estructuras de concreto reforzado expuestas a ambientes agresivos, han desarrollado concretos de alto desempeño que se caracterizan por sus bajas relaciones agua/cemento y el empleo de aditivos químicos y materiales suplementarios. Esto es con el propósito de reducir la permeabilidad e incrementar la durabilidad del concreto (Aïtcin, 2003; Mehta, 1999). El principal inconveniente de este tipo de concreto es su mayor costo de producción con respecto al concreto ordinario, debido al uso de una mayor cantidad de cemento, aditivos químicos especiales y los materiales suplementarios. Estos dos últimos no siempre están disponibles en el mercado local, por lo que el costo de transporte se incrementa considerablemente. Por tal razón se plantea la necesidad de otras alternativas de aditivos químicos de bajo costo para elaborar concretos con propiedades mecánicas y de durabilidad similares a las del concreto de alto desempeño.

Los aditivos orgánicos naturales han sido empleados en la producción de concreto y morteros por décadas, mejorando sus propiedades en estado fresco y endurecido. Por ejemplo, el mucílago de nopal ha sido utilizado como un agente aglutinante y modificador de viscosidad en la producción artesanal de morteros de cal y para trabajos de restauración de edificios históricos de adobe en América latina (Bishop y Funkhouser, 1998). Además, su uso en concreto hidráulico mejora la fluidez, retarda los tiempos de fraguado, facilita el manejo e incrementa la resistencia mecánica a edades tardías (Chandra et al., 1998; Ramírez-Arellanes et al, 2012). También se ha empleado el extracto de algas marinas en materiales base cemento, encontrando que retarda la hidratación del cemento, incrementa la resistencia mecánica y reduce la carbonatación y la permeabilidad a iones cloruro (Hernández et al, 2016).

A pesar de que se han realizado aplicaciones exitosas de estos aditivos en materiales base cemento, no existe información completa sobre cuáles son las modificaciones que se producen en la microestructura o en los productos de hidratación del cemento. El objetivo de este trabajo es conocer cómo influyen el mucílago de nopal y el extracto de algas cafés sobre el grado de hidratación de pastas de cemento con diferentes relaciones agua/cemento $(\mathrm{a} / \mathrm{c})$ y condiciones de curado.

\section{PROCEDIMIENTO EXPERIMENTAL}

\subsection{Materiales}

Se empleó cemento Portland ordinario CPO-30 RS, cuya composición química se muestra en la Tabla 1. Los aditivos naturales utilizados fueron el mucílago de nopal y el extracto de algas marinas cafés, cuya extracción y preparación se describe a continuación.

Mucilago de nopal: La extracción del mucílago consistió en mezclar en un recipiente rebanadas de nopal Opuntia ficus indica con agua destilada en proporción 1:1.5 en peso, a temperatura controlada no mayor de $60{ }^{\circ} \mathrm{C}$ durante tres horas y bajo agitación constante. El mucílago obtenido fue la fase acuosa resultante del filtrado progresivo a través de los tamices No. $16(1.18 \mathrm{~mm})$, No. $100(150 \mu \mathrm{m})$ y No. $200(75 \mu \mathrm{m})$. Con el propósito de incrementar la concentración del extracto de mucílago, éste se sometió a un proceso de ultrafiltración a través de un cartucho de fibra hueca de Polisulfona de $30 \mathrm{KDa}$. De este proceso se obtuvieron dos tipos de fases acuosas. La primera que fue la fase retenida denominada mucílago retenido (MR), la cual consistió principalmente de polisacáridos y fibras de micro celulosa. La segunda fase que fue denominada mucílago permeado (MP), consistió de azúcares libres y colorantes oxidados de bajo peso molecular. Las concentraciones del aditivo MR usadas en la elaboración de las pastas de cemento, fueron de $0.5 \%, 1 \%$ y $1.82 \%$ peso/volumen (p/v); y de $0.5 \%, 1 \%$ y $1.26 \%$ para el MP.

Extracto de algas marinas cafés: Se utilizó una dispersión acuosa concentrada de algas cafés molidas de Macrocytis pyrifera para preparar soluciones de extracto de algas a distintas concentraciones. El

Nexo Revista Científica / Vol. 30, No. 02, pp. 73-83 / Diciembre 2017 
concentrado inicial se mezcló con agua destilada en relación 1:1 en volumen y se agitó de manera constante hasta obtener una dispersión homogénea. Esta se calentó a una temperatura de $40^{\circ} \mathrm{C}$ durante 5 minutos, con el propósito de facilitar su filtración. Los tamices empleados progresivamente durante el proceso de filtración fueron los No. $30(0.59 \mathrm{~mm})$, No. $50(300 \mu \mathrm{m})$ y No. $100(150 \mu \mathrm{m})$. Las concentraciones de extracto de algas (A) usadas en la elaboración de las pastas de cemento fueron de $0.5 \%, 1 \%$ y $1.82 \%$ (p/v).

Tabla 1. Composición química del cemento CPO-30 RS

\begin{tabular}{cc}
\hline Componentes & \% en masa \\
\hline $\mathrm{SiO}_{2}$ & 18.77 \\
$\mathrm{Al}_{2} \mathrm{O}_{3}$ & 3.69 \\
$\mathrm{Fe}_{2} \mathrm{O}_{3}$ & 3.97 \\
$\mathrm{CaO}$ & 58.77 \\
$\mathrm{MgO}$ & 1.58 \\
$\mathrm{~K}_{2} \mathrm{O}+\mathrm{Na}_{2} \mathrm{O}$ & 0.49 \\
$\mathrm{MnO}$ & 0.10 \\
$\mathrm{P}_{2} \mathrm{O}_{5}$ & 0.10 \\
$\mathrm{TiO}_{2}$ & 0.17 \\
$\mathrm{SO}_{3}$ & 2.54 \\
$\mathrm{PXC}$ & 5.39 \\
\hline
\end{tabular}

\subsection{Especímenes de pasta de cemento}

Se prepararon pastas de cemento de acuerdo con la norma ASTM C 305 (ASTM, 1999), con relaciones a/c en peso de 0.30 y 0.60 (Tabla 2). Para lograr una mezcla homogénea, se empleó una batidora de movimiento planetario. Los especímenes fueron colados en moldes cilíndricos de $40 \mathrm{~mm}$ de diámetro por $80 \mathrm{~mm}$ de altura. Los tipos de curado fueron sellado y en húmedo hasta la edad de prueba.

El curado en húmedo consistió en almacenar los especímenes en agua saturada con hidróxido de calcio $(0.5$ $\%$ en peso) a temperatura ambiente. El curado sellado consistió en mantener la pasta de cemento dentro de los recipientes de plástico en que fueron colados, cerrados con una tapa plástica hasta la edad de prueba. El grado de hidratación de las pastas se determinó por medio de la técnica de pérdida de masa por ignición y análisis termogravimétrico (ATG), a los 14, 28 y 56 días.

Tabla 2. Pastas de cemento

\begin{tabular}{cccc}
\hline \multirow{2}{*}{$\begin{array}{c}\text { Relación } \\
\mathrm{a} / \mathrm{c}\end{array}$} & Aditivos naturales & $\begin{array}{c}\text { Concentración de aditivos }(\% \\
\mathrm{p} / \mathrm{v})\end{array}$ & $\begin{array}{c}\text { Concentración de } \\
\text { Aditivos con respecto a la masa } \\
\text { de cemento }(\% \mathrm{p} / \mathrm{p})\end{array}$ \\
\hline \multirow{3}{*}{0.30} & Control & Ninguna & \\
\cline { 2 - 4 } & Mucílago retenido (MR) & $0.5,1 \mathrm{y} 1.82$ & $0.15,0.30 \mathrm{y} 0.56$ \\
\cline { 2 - 4 } & Mucílago permeado (MP) & $0.5,1 \mathrm{y} 1.26$ & $0.15,0.30 \mathrm{y} 0.38$ \\
\cline { 2 - 4 } 0.60 & Extracto de alga (A) & $0.5,1 \mathrm{y} 1.82$ & $0.15,0.30 \mathrm{y} 0.56$ \\
\cline { 2 - 4 } & Control & Ninguna & $0.30,0.60 \mathrm{y} 1.11$ \\
\cline { 2 - 4 } & Mucílago retenido (MR) & $0.5,1 \mathrm{y} 1.82$ & $0.30,0.60 \mathrm{y} 0.77$ \\
\cline { 2 - 4 } & Mucílago permeado (MP) & $0.5,1 \mathrm{y} 1.26$ & $0.30,0.60 \mathrm{y} 1.11$ \\
\hline
\end{tabular}




\section{E.F. Hernández et al.}

3.3 Determinación del grado de hidratación por ignición y por análisis termogravimétrico (ATG)

La técnica de pérdida de masa por ignición se empleó para determinar el contenido de agua químicamente combinada en las pastas de cemento. A las edades establecidas, se trituraron y molieron muestras de pastas de cemento agregando acetona grado analítico hasta obtener un polvo fino, el cual se mantuvo en reposo en acetona durante 24 horas con el propósito de detener la hidratación del cemento. Posteriormente se secaron a $105^{\circ} \mathrm{C}$ hasta alcanzar masa constante. La cantidad de agua químicamente combinada $\left(\mathrm{W}_{\mathrm{n}}\right)$ de cada muestra fue la pérdida de masa entre $105^{\circ} \mathrm{C}$ y $1000{ }^{\circ} \mathrm{C}$, dividido por la masa de la muestra calcinada. La masa de la pasta calcinada se corrigió por la pérdida de masa por ignición del cemento anhidro (Lam et al., 2000).

El grado hidratación $\alpha(\%)$ de la pasta de cemento se determinó de acuerdo con (1).

$$
\alpha=100 \cdot \frac{w_{n}}{0.23}
$$

El análisis termogravimétrico (ATG) se empleó para determinar las cantidades de $\mathrm{W}_{\mathrm{n}} \mathrm{y}$ de hidróxido de calcio $(\mathrm{CH})$ a partir de polvo de pastas de cemento extraído a los 14, 28 y 56 días de edad. La preparación de las muestras consistió en triturar las pastas de cemento hasta obtener un polvo fino, el cual se secó a 50 ${ }^{\circ} \mathrm{C}$ por 48 horas. El ATG se realizó utilizando un equipo TA Instruments Q500 (TA Instruments, New Castle, DE, USA). La descomposición se realizó bajo una atmosfera de nitrógeno, empleando una rampa de calentamiento de $20{ }^{\circ} \mathrm{C} /$ min desde $30{ }^{\circ} \mathrm{C}$ hasta $900{ }^{\circ} \mathrm{C}$. Los valores de $\mathrm{W}_{\mathrm{n}}$ y CH fueron obtenidos a partir de la pérdida de masa de las muestras entre $105^{\circ} \mathrm{C}$ y $900{ }^{\circ} \mathrm{C}$, de acuerdo con (2) (Lam et al., 2000; Li et al., 2009).

$$
W_{n}=\frac{\mathrm{m}_{900}-\mathrm{m}_{105}}{\mathrm{~m}_{900}}-\left(\mathrm{f}_{\mathrm{c}} * \mathrm{LOI}\right)
$$

donde $\mathrm{m}_{105}$ y $\mathrm{m}_{900}$ son las masas de la muestra a $105^{\circ} \mathrm{C}$ y $900{ }^{\circ} \mathrm{C}$, respectivamente, $\mathrm{f}_{\mathrm{c}}$ es la fracción de masa de cemento, y LOI es el \% de pérdida de masa por ignición del cemento anhidro.

De acuerdo con El-Jazairi \& Illston (1977), la descomposición del $\mathrm{CH}$ ocurre entre $400{ }^{\circ} \mathrm{C}$ y $600{ }^{\circ} \mathrm{C}$ de la siguiente manera:

$$
\mathrm{Ca}(\mathrm{OH})_{2} \longrightarrow \mathrm{CaO}+\mathrm{H}_{2} \mathrm{O}
$$

Por lo tanto, el contenido de CH, se determinó de acuerdo con (3) (Justnes et al., 1998):

$$
\mathrm{CH}=\frac{74.09}{18.01} \cdot \frac{\mathrm{m}_{400}(\mathrm{t})-\mathrm{m}_{600}(\mathrm{t})}{\mathrm{m}_{900}}
$$

donde $\mathrm{m}_{400}(\mathrm{t})$ es el peso de la muestra a $400{ }^{\circ} \mathrm{C}$ a un tiempo $\mathrm{t}, \mathrm{m}_{600}(\mathrm{t})$ es el peso de la muestra a $600{ }^{\circ} \mathrm{C}$ a un tiempo $t$, $\mathrm{m}_{900}$ es el peso de la muestra a $900{ }^{\circ} \mathrm{C}, 74.09 / 18.01$ es la relación de masa molar de $\mathrm{Ca}(\mathrm{OH})_{2} /$ $\mathrm{H}_{2} \mathrm{O}$.

Una vez determinadas $\mathrm{W}_{\mathrm{n}}$ y CH, se calculó el grado de hidratación a partir de (4) y (5).

$$
\alpha_{\mathrm{Wn}}=\frac{\mathrm{w}_{n}}{\mathrm{w}_{n}(\infty)}
$$




$$
\alpha_{\mathrm{CH}}=\frac{\mathrm{CH}}{\mathrm{CH}(\infty)}
$$

donde $\mathrm{W}_{\mathrm{n}}(\infty)=0.27$ y $\mathrm{CH}(\infty)=0.29$

\section{RESULTADOS Y DISCUSIÓN}

En la Figura 1, se muestra el grado de hidratación de las pastas de cemento con relación a/c $=0.30$, con curado sellado y curado en húmedo, a los 28 y 56 días de edad. En la Figura 1a se observa, como se esperaba, que el curado en húmedo incrementa el grado de hidratación de las pastas de cemento con respecto al curado sellado. El agua adicional aportada por el curado en húmedo contribuye a incrementar las reacciones químicas con el cemento sin hidratar.

Con respecto a la influencia de las adiciones orgánicas en el grado de hidratación de las pastas de cemento, a los 28 días se observa que las pastas con adiciones orgánicas presentan grados de hidratación mayores que el control. A los 56 días, únicamente en las pastas MR0.56 con curado sellado, el grado de hidratación fue mayor con respecto al control.

En la Figura $1 \mathrm{~b}$ se observa un incremento en el grado de hidratación de las pastas MP a los 28 días de edad con respecto al control. A los 56 días se observa que el grado de hidratación es mayor que el control en las pastas MP0.30 con curado sellado. Este comportamiento puede estar relacionado con la capacidad del mucílago de nopal para retener agua, funcionando como agentes de curado interno que liberan el agua retenida para hidratar el cemento (Chandra et al., 1998; Ramírez-Arellanes et al., 2012).

Para el caso de las pastas conteniendo extracto de algas (Figura 1c), el incremento del grado de hidratación observado a los 28 días de edad, puede deberse principalmente a su tendencia a formar geles con los iones de calcio presentes en las pastas de cemento (Pathak et al., 2010). A pesar de esta capacidad de formar geles, el efecto de curado interno observado fue diferente al observado en las pastas que contenían mucílago de nopal.

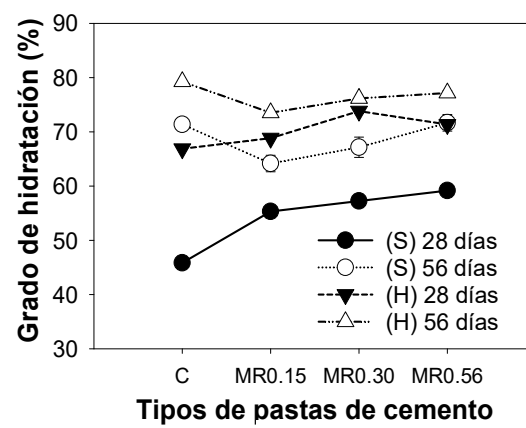

(a)

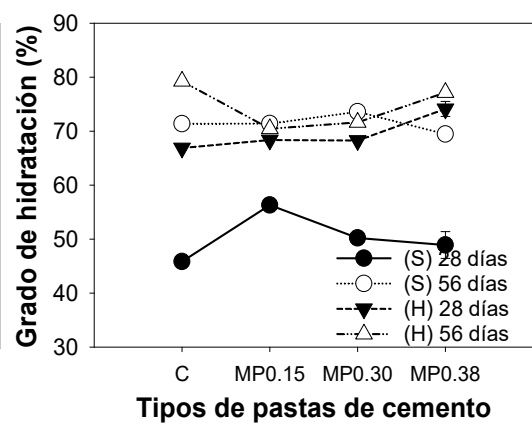

(b)

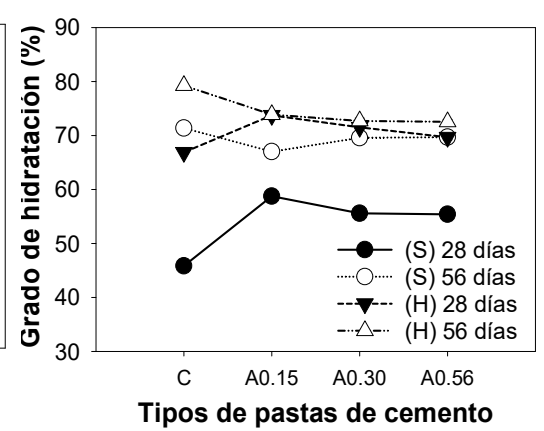

(c)

Figura 1. Grado de hidratación de pastas de cemento con relación a/c $=0.30$, con curado sellado $(\mathrm{S})$ y húmedo $(\mathrm{H})$.

a) mucílago retenido (MR), b) mucílago permeado (MP) y c) extracto de algas (A)

En la Figura 2 se presenta el grado de hidratación de las pastas de cemento con relación a/c $=0.60$, con curado sellado y húmedo, a los 28 y 56 días de edad. En general, se observa también que el tipo de curado en húmedo produce un incremento en el grado de hidratación en comparación con el curado sellado. Con respecto a la influencia del tipo de aditivo, se observa que a los 28 días en las pastas que contenían MR y MP a la mayor concentración, el grado de hidratación se redujo drásticamente con respecto al control. Para el caso de las pastas que contenían extracto de algas, a los 28 días de edad se observa que el grado de hidratación incrementa a medida que aumenta la concentración. Esto último, como consecuencia de la 
capacidad de estos aditivos de retener agua y de formar compuestos entre el polímero con los iones divalentes de $\mathrm{Ca}^{2+}$ presentes en las pastas de cemento (Pathak et al., 2010). A los 56 días, únicamente en las pastas MR con la mayor concentración del aditivo se observa inhibición de la hidratación del cemento con ambos tipos de curado, con respecto al control. Este efecto retardante puede deberse a la adsorción de estos aditivos en los primeros hidratos formados durante la hidratación temprana, generando un recubrimiento que limita la velocidad de reacción del cemento con el agua (Hernández et al, 2016).

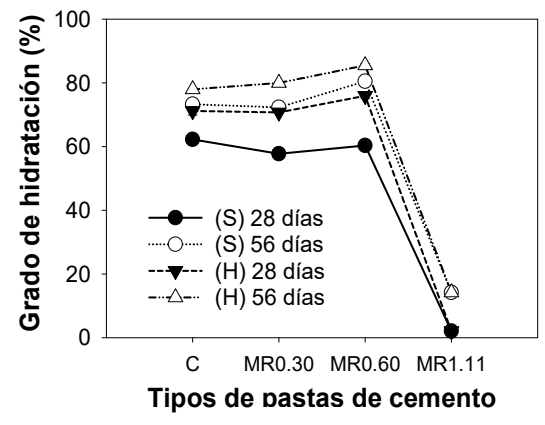

(a)

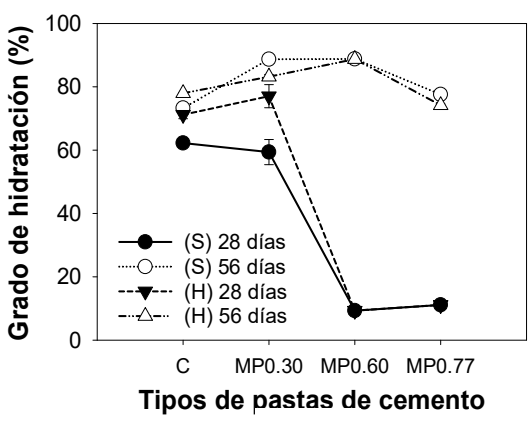

(b)

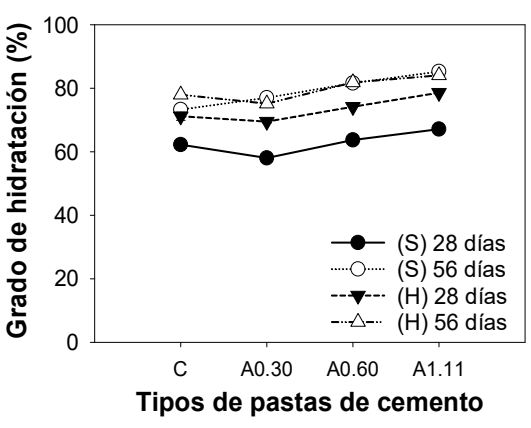

(c)

Figura 2. Grado de hidratación de pastas de cemento con relación a / c $=0.60$, con curado sellado $(\mathrm{S})$ y húmedo $(\mathrm{H})$. a) mucílago retenido (MR), b) mucílago permeado (MP) y c) extracto de algas (A)

En la Figura 3 se ejemplifica los resultados del análisis termogravimétrico de las pastas de cemento conteniendo mucílago de nopal, con relación a/c $=0.30$ y curado sellado a los 56 días de edad. Se observa que las pastas control son las que presentan mayor pérdida de masa con respecto a las que contienen mucílago de nopal, por lo tanto, el contenido de $\mathrm{W}_{\mathrm{n}} \mathrm{y}$ el grado de hidratación es mayor. En la derivada de la masa se observa la pérdida del agua evaporable $\left(30-105^{\circ} \mathrm{C}\right)$, la descomposición del yeso y etringita $(110$ $\left.-170^{\circ} \mathrm{C}\right)$, el hidróxido de calcio entre $400{ }^{\circ} \mathrm{C}-600^{\circ} \mathrm{C}$, los silicatos hidratados de calcio $\left(180{ }^{\circ} \mathrm{C}-300{ }^{\circ} \mathrm{C}\right)$ y la de carbonato de calcio $\left(700^{\circ} \mathrm{C}-900^{\circ} \mathrm{C}\right)$ (El-Jazairi \& Illston, 1977; Noumowé, 1995).
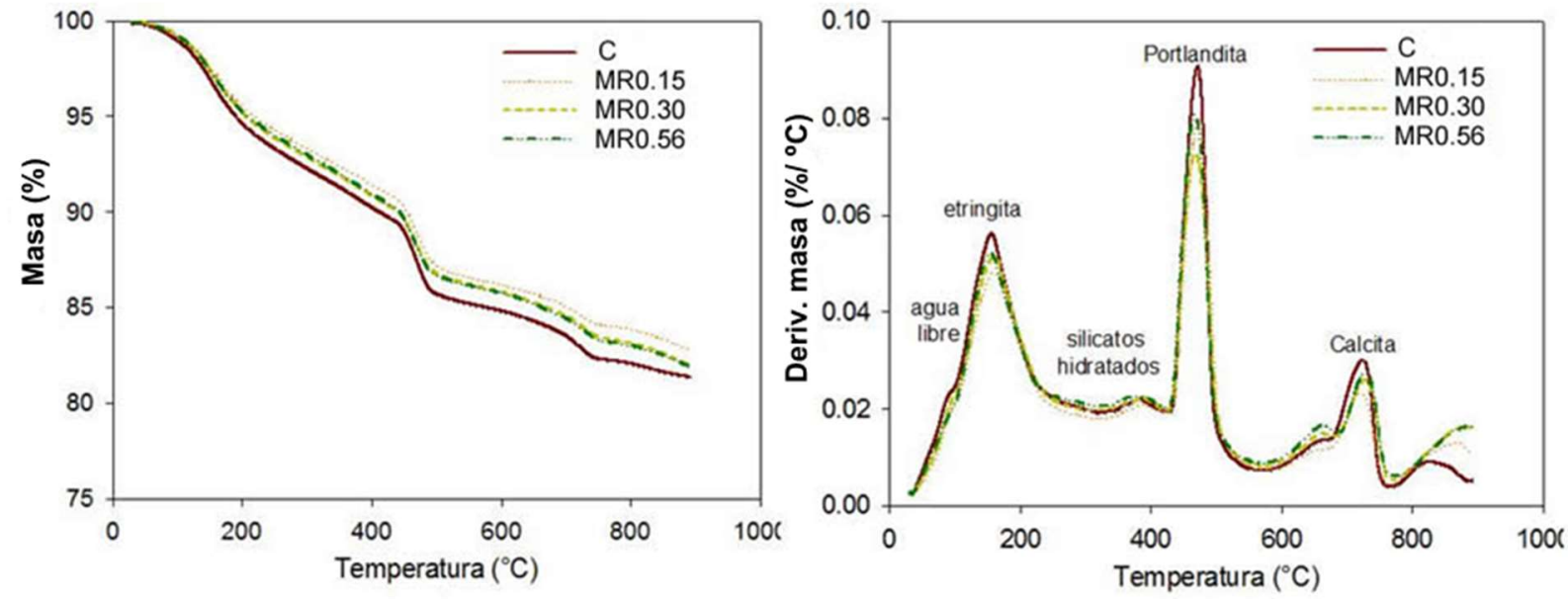

Figura 3. Resultados de ATG de pastas de cemento conteniendo mucílago de nopal, con relación a/c $=0.30$, a 56 días de edad, curado sellado

Con base en los resultados de descomposición por temperatura obtenidos de ATG, se determinaron las cantidades de $\mathrm{W}_{\mathrm{n}}$ y $\mathrm{CH}$ para cada tipo de mezcla a los 14, 28 y 56 días de edad y se calculó el grado de hidratación. En la Figura 4, se observa que el contenido de $\mathrm{W}_{\mathrm{n}}$ de las pastas de cemento con relación a/c $=$ 0.30 conteniendo adiciones orgánicas y con curado sellado, no muestran una tendencia clara de 
comportamiento con respecto a la edad. Por el contrario, se observa un incremento en el contenido de $\mathrm{CH}$ en función del tiempo. A los 28 días, el contenido de $\mathrm{W}_{\mathrm{n}} \mathrm{y} \mathrm{CH}$ en las pastas con adiciones orgánicas fueron mayores con respecto al control. Mientras que, a los 56 días de edad, muestran valores similares al control. De manera general, se observa que el comportamiento del contenido de $\mathrm{W}_{\mathrm{n}} \mathrm{y} \mathrm{CH}$ en las pastas de cemento, fue similar al obtenido por la técnica de pérdida de masa por ignición.

En la Figura 5, se muestra el contenido de $\mathrm{W}_{\mathrm{n}}$ y $\mathrm{CH}$, a los 14 y 28 días de edad, de las pastas con relación $\mathrm{a} / \mathrm{c}=0.30$, curado en húmedo. A los 14 días las pastas que contenían adiciones orgánicas presentan valores menores de $\mathrm{W}_{\mathrm{n}}$ con respecto al control, con excepción de la mezcla A0.15. A los 28 días en todas las pastas que contenían adiciones, los valores de $\mathrm{W}_{\mathrm{n}} \mathrm{y} \mathrm{CH}$ fueron mayores con respecto al control. Se observa que estos valores incrementan al aumentar la concentración de los aditivos.

En la Figura 6 y 7 , se presenta el contenido de $\mathrm{W}_{\mathrm{n}}$ y $\mathrm{CH}$ de pastas de cemento con a/c $=0.60$, con curado sellado y curado húmedo. Los resultados del contenido de $\mathrm{W}_{\mathrm{n}}$ en las pastas con curado en húmedo no muestran una tendencia de incremento con respecto a la edad. La tendencia de los valores de $\mathrm{W}_{\mathrm{n}} \mathrm{y} \mathrm{CH}$, son congruentes con los resultados obtenidos de grado de hidratación por la técnica de ignición. En las tres edades de prueba se observa que las pastas que contenían adiciones orgánicas presentan valores mayores de contenido de $\mathrm{W}_{\mathrm{n}}$ y $\mathrm{CH}$, con respecto al control, con excepción de las mezclas MR1.11.
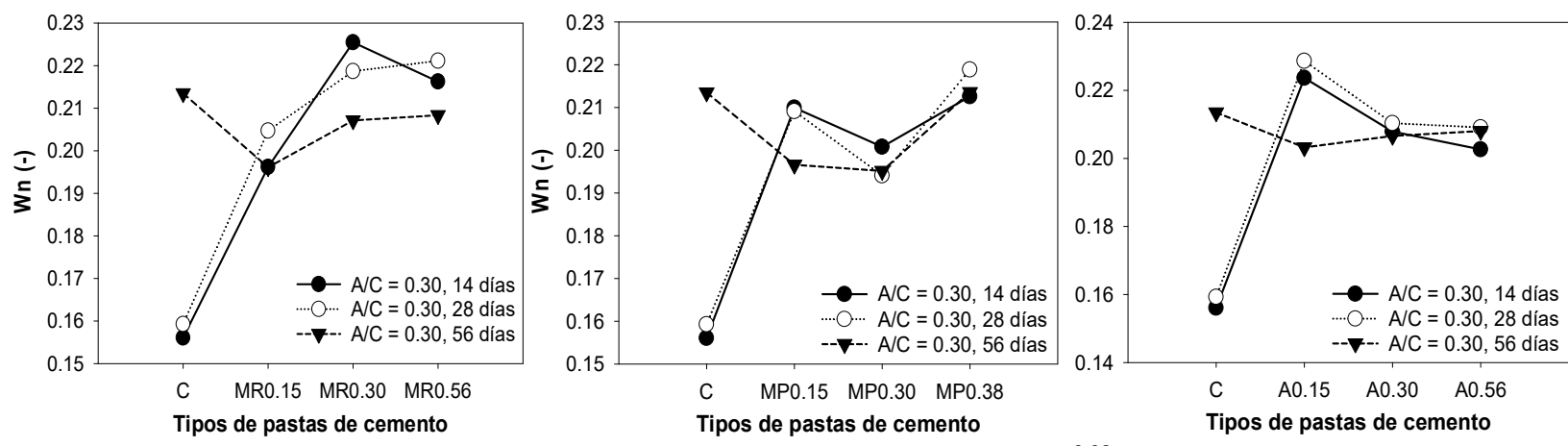

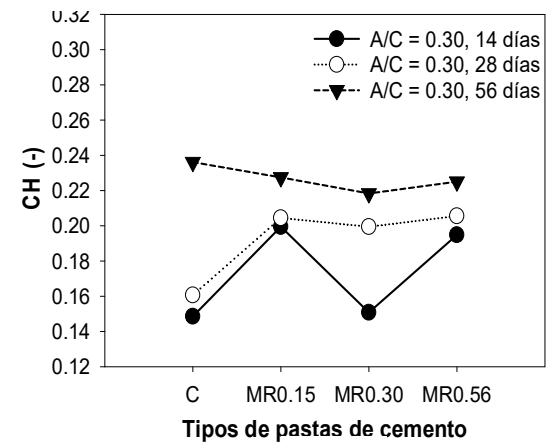

(a)

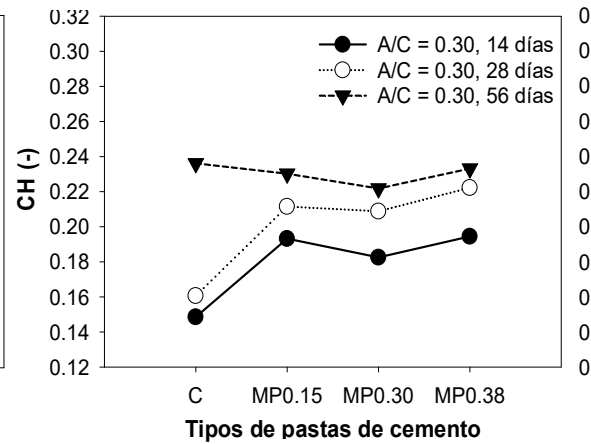

(b)

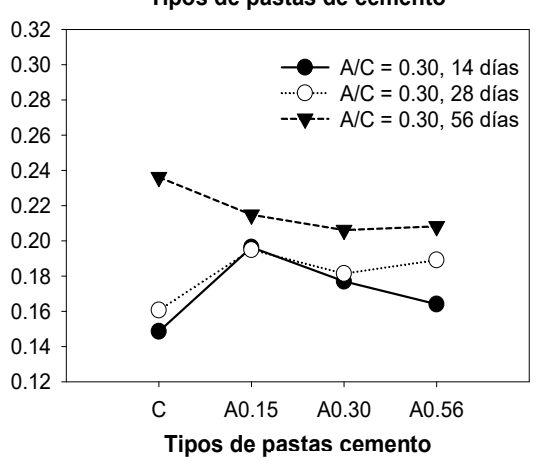

(c)

Figura 4. Contenido de Wn y $\mathrm{CH}$ en pastas de cemento con relación a/c $=0.30$, a 14, 28 y 56 días de edad, curado sellado. a) mucílago retenido (MR), b) mucílago permeado (MP) y c) extracto de algas (A) 

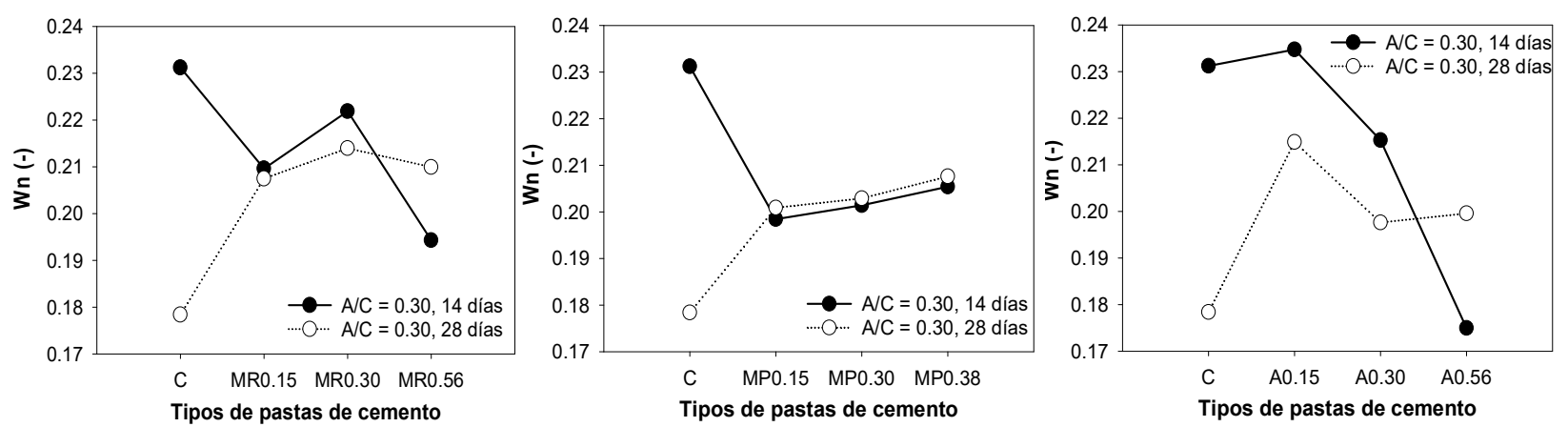

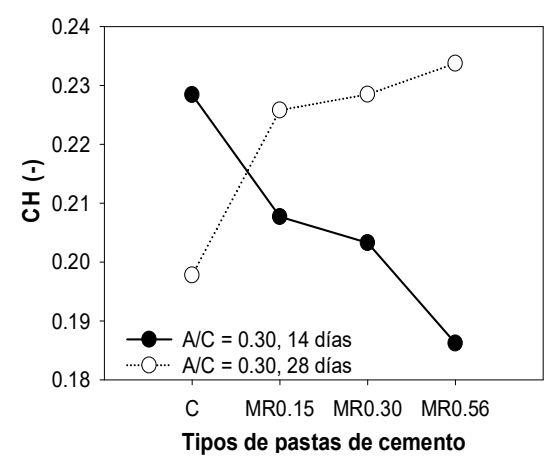

(a)

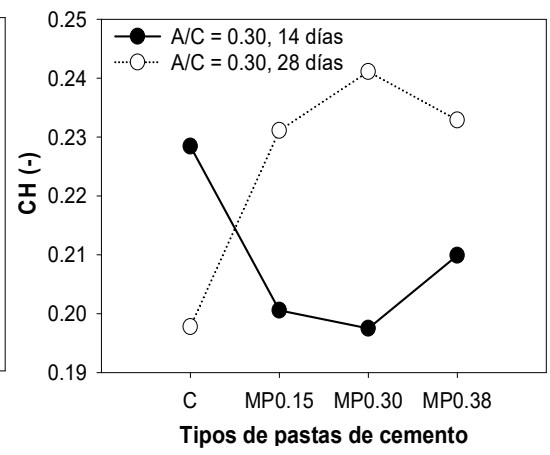

(b)

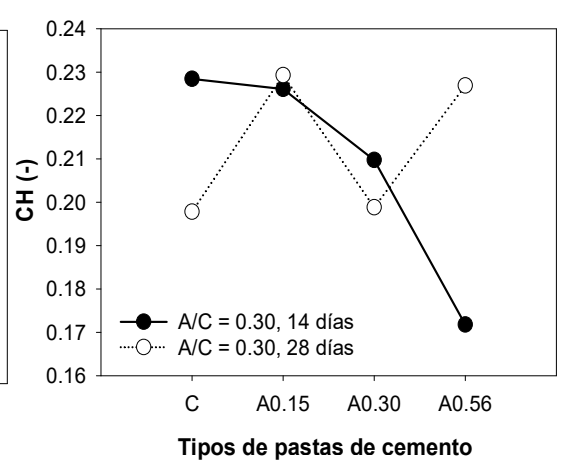

(c)

Figura 5. Contenido de $\mathrm{Wn}$ y $\mathrm{CH}$ en pastas de cemento con relación a/c $=0.30$, a 14 y 28 días de edad, curado en húmedo. a) mucílago retenido (MR), b) mucílago permeado (MP) y c) extracto de algas (A)
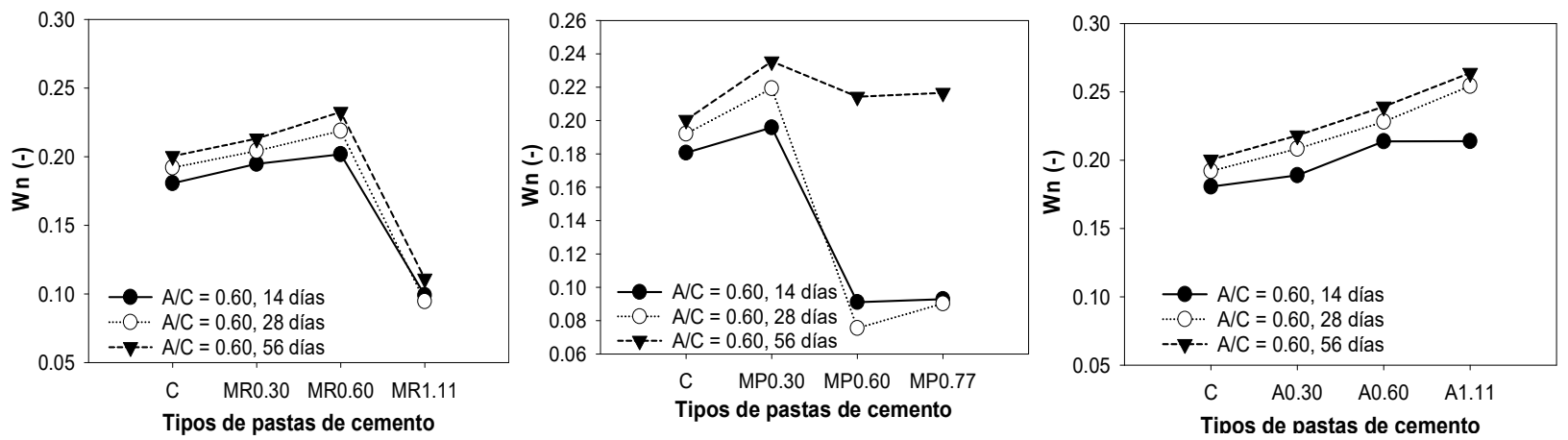

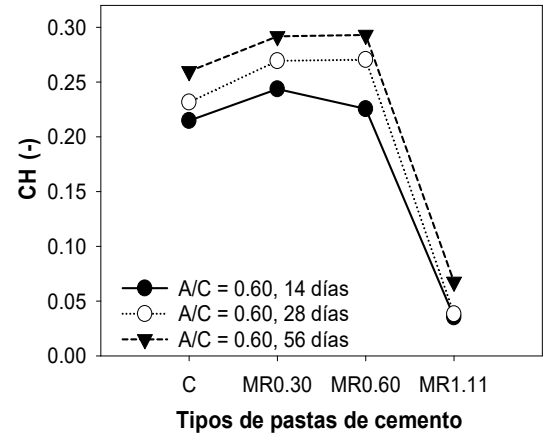

(a)

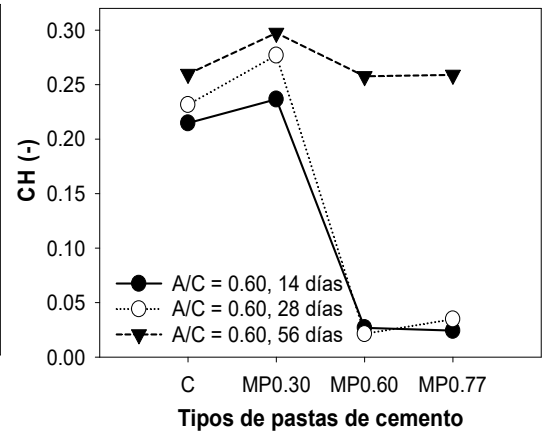

(b)

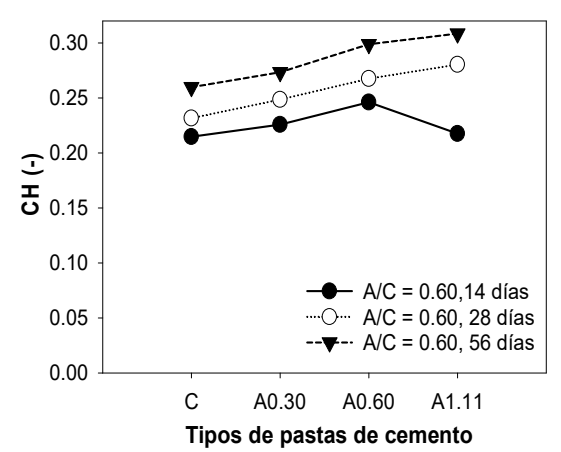

(c)

Figura 6. Contenido de Wn y CH, en pastas de cemento con relación a / c $=0.60$, a 14, 28 y 56 días de edad, curado sellado. a) mucílago retenido (MR), b) mucílago permeado (MP) y c) extracto de algas (A) 

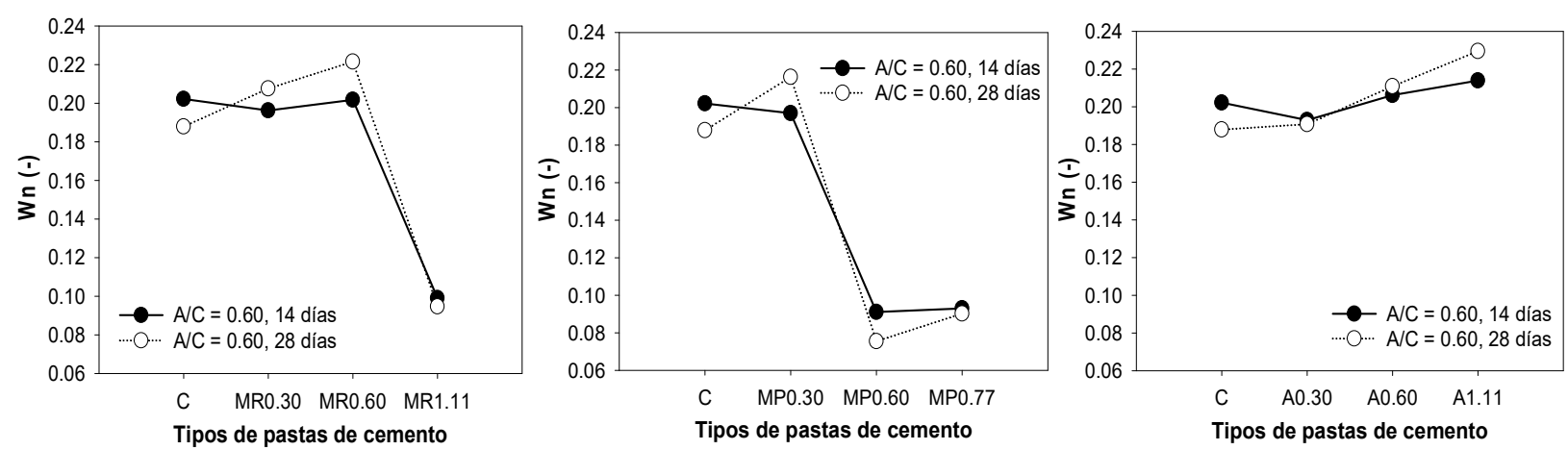

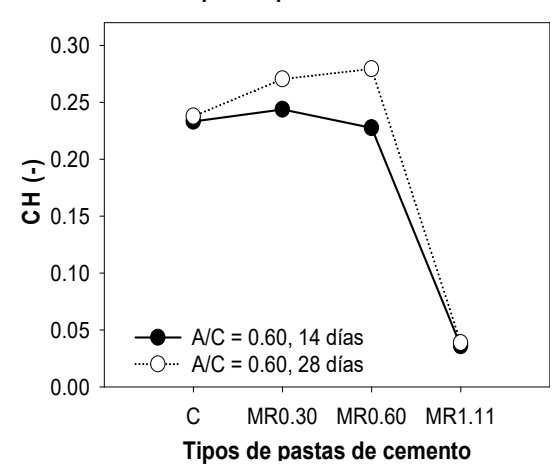

(a)

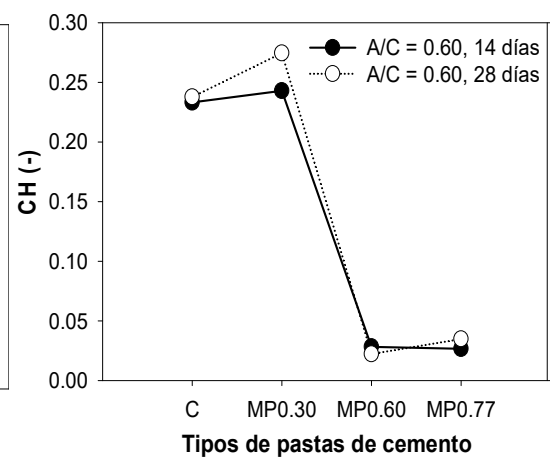

(b)

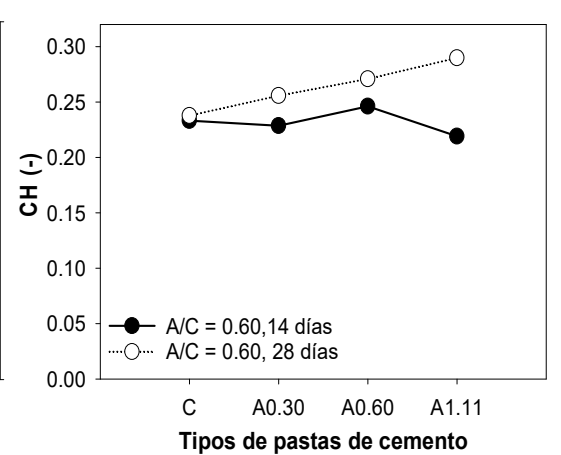

(c)

Figura 7. Contenido de $\mathrm{W}_{\mathrm{n}} \mathrm{y} \mathrm{CH}$ en pastas de cemento con relación a/c $=0.60$, a 14 y 28 días de edad, curado en húmedo. a) mucílago retenido (MR), b) mucílago permeado (MP) y c) extracto de algas (A)

Con los valores de $\mathrm{W}_{\mathrm{n}} \mathrm{y} \mathrm{CH}$, se determinó el grado de hidratación de las pastas con curado sellado a los 28 días de edad. Los valores de grado de hidratación obtenidos por ATG para Wn y $\mathrm{CH}$, fueron correlacionados con los valores de grado de hidratación obtenidos con la técnica de pérdida de masa por ignición (Figura 8). Se observa que el coeficiente de determinación $\mathrm{R}^{2}$ está por encima de 0.90 . De este resultado, los valores de grado de hidratación obtenidos con $\mathrm{Wn}$, son los que muestran una mejor correlación con respecto al grado de hidratación obtenido por la técnica de ignición. Con base en estos resultados, se debe considerar la técnica de análisis termogravimétrico como una herramienta adecuada para evaluar el grado de hidratación de pastas de cemento.

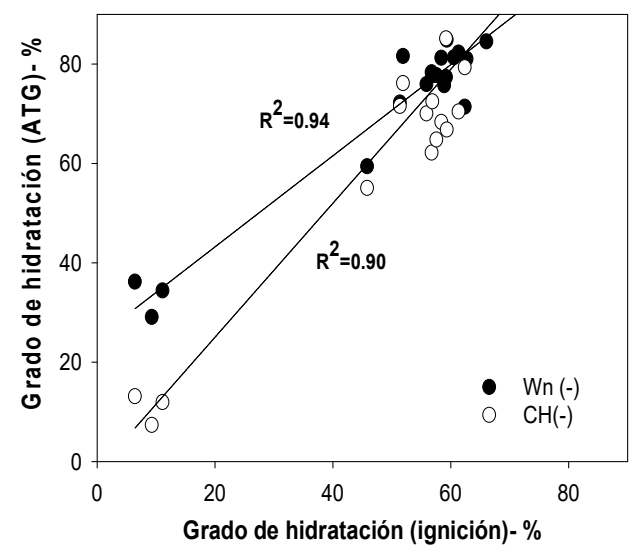

Figura 8. Correlación del grado de hidratación de pastas de cemento por la técnica de pérdida de masa por ignición, con grado de hidratación por ATG para valores de $\mathrm{W}_{\mathrm{n}}$ y CH. La Figura incluye las pastas de cemento con relaciones $\mathrm{a} / \mathrm{c}=0.30$ y 0.60 , curado sellado, a 28 días de edad 


\section{CONCLUSIONES}

Con base en los resultados experimentales se concluye que:

- El grado de hidratación de las pastas de cemento con relación a/c $=0.30$ conteniendo adiciones orgánicas fue mayor con respecto al control únicamente en las pastas MP0.30. En las pastas de cemento con relación a/c $=0.60$ conteniendo adiciones orgánicas, el grado de hidratación fue mayor con respecto al control, con excepción de las muestras MR1.11, las cuales presentaron un severo retardo hasta los 56 días de edad.

- El incremento del grado de hidratación observado en las pastas de cemento que contenían adiciones orgánicas en comparación con el control, puede estar relacionado con la propiedad de estos polímeros de retener agua, lo cual limita la disponibilidad de agua para las reacciones de hidratación en comparación con las de control. El agua retenida puede ser liberada a diferentes edades, reaccionando con el cemento e incrementando el grado de hidratación.

- El efecto retardante del mucílago de nopal sobre el fraguado del cemento, es una propiedad que puede ser aprovechada en la elaboración de concreto en climas cálidos para mantener la trabajabilidad durante un mayor tiempo.

\section{REFERENCIAS}

Aïtcin, P. C. (2003). The durability characteristics of high performance concrete: a review. Cement and Concrete Composites, 25 (4-5), 409-420.

Mehta, P. K. (1999). Advancements in concrete technology. Concrete International, 21 (6), 69-76.

Bishop, M. H., Funkhouser, V. M. (1998). A consideration of the ethnobotany of nopal (Opuntia spp) and conservation implications. WAAC annual meeting, Western association of art conservation.

Chandra, S., Eklund, L., Villareal, R. R. (1998). Use de cactus in mortars and concrete. Cement and concrete research, $28(1), 41-51$.

Ramírez-Arellanes, S., Cano-Barrita, P. F. de J., Julián-Caballero, F., Gómez-Yáñez, C. (2012). Propiedades de durabilidad en hormigón y análisis microestructural en pastas de cemento con adición de mucílago de nopal como aditivo natural. Materiales de Construcción, 62 (302), 327-341.

Hernández, E. F., Cano-Barrita, P. F. de J., Torres-Acosta, A. A. (2016). Influence of cactus mucilage and marine brown algae extract on the compressive strength and durability of concrete. Materiales de Construcción, 66 (321).

American Society for Testing Materials (ASTM). (1999). ASTM Standard C305-99: Standard practice for mechanical mixing of hydraulic cement pastes and mortars of plastic consistency. West Conshohocken, PA, 3. 
Lam, L., Wong, Y. L., Poon, C. S. (2000). Degree of hydration and gel/space ratio of high-volume fly ash/cement systems. Cement and concrete research, 30 (5), 747-756.

Li, X., Yan, PY., A, RH. (2009). Assessment method of hydration degree of cement in complex binder based on the calcium hydroxide content (in Chinese). J Chin. Ceram. Soc., 137, 1597-61.

El-Jazairi B., Illston J.M. (1977). A simultaneous semi-isothermal method of thermogravimetry and derivative thermogravimetry, and its application to cement pastes. Cem. Concr. Res., 7, 247-258.

Justnes, H., Sellevold, E. J., Reyniers, B., Van Loo, D., Van Gemert, A., Verboven, F., Van Gemert, D. (1998). The influence of cement characteristics on chemical shrinkage, in: E.-i. Tazawa (Ed.), Proceedings of the International Workshop on Autogenous Shrinkage of Concrete AUTO-SHRINK'98, E \& FN Spon, London, UK, 71-80.

Pathak, T. S., Yun, J. H., Lee, J., Paeng, K. J. (2010). Effect of calcium ion (cross-linker) concentration on porosity, surface morphology and thermal behavior of calcium alginates prepared from algae (Undaria pinnatífida). Carbohydrate Polymers, 81 (3), 633-639.

Noumowé A. (1995). Effet des hautes températures $\left(20 \mathrm{jC}-600{ }^{\circ} \mathrm{C}\right)$ sur le béton. $\mathrm{PhD}$ thesis, Institut National des Sciences Appliquées.

\section{SEMBLANZA DE LOS AUTORES}

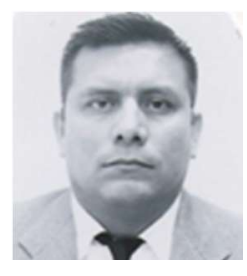

Eddisson Francisco Hernández: Obtuvo el grado de Arquitecto en la Universidad Nacional de Ingeniería, Nicaragua. Obtuvo los grados de maestría y doctorado en el Instituto Politécnico Nacional, México. Ha realizado estancias de investigación en la Universidad Nacional Autónoma de México y en la Universidad Marista de Querétaro, México. Sus intereses de investigación incluyen la ciencia y tecnología de materiales base cemento, así como la corrosión del acero de refuerzo en estructuras de concreto. Actualmente es investigador de la Universidad Nacional de Ingeniería, Nicaragua.

Heriberto Pfeiffer Perea: Doctor en Química de la Universidad Autónoma Metropolitana, Iztapalapa. Ha realizado estancias posdoctorales en el Departamento de Materiales y Metalurgia de la Universidad de Cambridge, Inglaterra; y en la Escuela Politécnica de la Universidad de Nantes, Francia. Es investigador Titular C del Instituto de Investigaciones en Materiales de la Universidad Nacional Autónoma de México. Actualmente es Coordinador del Posgrado en Ciencia e Ingeniería de Materiales. Es investigador nivel III del sistema nacional de investigadores (SNICONACYT) de México.

Prisciliano Felipe de Jesús Cano Barrita: Doctor en Ingeniería Civil, Universidad de New Brunswick, Canadá. Sus intereses de investigación incluyen la ciencia y tecnología de materiales base cemento y técnicas no destructivas para la caracterización de materiales. Actualmente es investigador del CIIDIR Unidad Oaxaca del Instituto Politécnico Nacional, Investigador nivel I del sistema nacional de investigadores (SNI-CONACYT) de México. 